\title{
Autonomic function test during the COVID-19 pandemic: the Stanford experience
}

\author{
Dong In Sinn ${ }^{1} \circledast$. Srikanth Muppidi ${ }^{1} \cdot$ Mitchell G. Miglis $^{1} \cdot$ Safwan Jaradeh $^{1}$
}

Received: 22 September 2020 / Accepted: 2 December 2020 / Published online: 2 January 2021

(c) Springer-Verlag GmbH, DE part of Springer Nature 2021

Keywords Autonomic function test · COVID-19 - Safety protocol

Dear Editors,

Recently, the American Autonomic Society (AAS) released a position statement, providing guidance for safely resuming autonomic function tests (AFTs) during the coronavirus disease 2019 (COVID-19) pandemic [1]. When mandatory shelter-at-home orders were issued by local governments in March 2020, our autonomic center began developing its own AFT safety protocol pursuant to institutional policy and the recommendations from the Centers for Disease Control and Prevention (CDC) [2]. When both personal protective equipment (PPE) supply and COVID-19 polymerase chain reaction (PCR) test availability improved, the center, armed with our safety protocol, felt confident enough to gradually resume AFTs. We would like to share our experience from implementing such protocol.

Our current safety protocol is as follows:

All AFTs are scheduled in advance. Two weeks before the AFT, a nurse contacts the patient to convey information regarding institutional safety protocol and the requirement that the patient must receive a negative COVID-19 PCR report no earlier than 7 days prior to the AFT. A technician contacts the patient to reaffirm the requirement.

Only one entrance of our facility is open, where clinic staff screen each patient and health care worker (HCW) for COVID-19 symptoms. They receive new surgical masks. They must cover the mouth and the nostrils. A family member can accompany a patient only when the patient requires assistance. A technician accompanies a patient from the waiting area.

Dong In Sinn

dsinn@stanford.edu

1 Department of Neurology, Stanford Autonomic Disorders Program, Stanford University, Palo Alto, California, USA
HCWs must wear surgical masks, protective eye (or facial) shields and disposable gloves during AFT. Wearing a disposable gown is not mandatory. Patients must keep the surgical masks on, except during thermoregulatory sweat test (TST) and Valsalva maneuver (VM). During TST, the patient wears an N95 mask while indicator powder is applied, and a surgical mask may be used afterwards. For VM, a single-use mouthpiece with a bacterial/viral filter is used. If the first attempt of deep breathing or VM shows unequivocally normal findings, the technician may move forward to the next test at his/her discretion. Other single-use items include tubes used during VM and capsules used for quantitative sudomotor tests.

A technician is permitted to leave the suite when a physician enters for a tilt-table test. When possible, physical distance is encouraged as recommended by the CDC [2]. All surfaces that the patient may contact are cleansed thoroughly between patients. There are no specific parameters regarding the adequate amount of time necessary for air change in the suites between tests.

For quality improvement and assurance, performance data between March 16 and August 21 were analyzed. No patients were denied entrance due to COVID-19 symptoms. A total of 267 patients had AFTs during the period, and 201 (75.3\%) visited the center on or after the week of May 11, when our protocol began to require the COVID-19 PCR test. Initially, PCR screening no earlier than 3 days before AFT was recommended only for VM. If a patient was unable to obtain PCR screening, the protocol permitted the performance of VM with additional PPE. There were two major incidents that caused our center to refine the protocol. The first incident involved an urgent PCR test on the same day as AFT. Its negative result, however, become available the following day. The second incident involved a patient who received a PCR test at a different facility 4 days prior. The test result was positive, but neither the patient nor the 


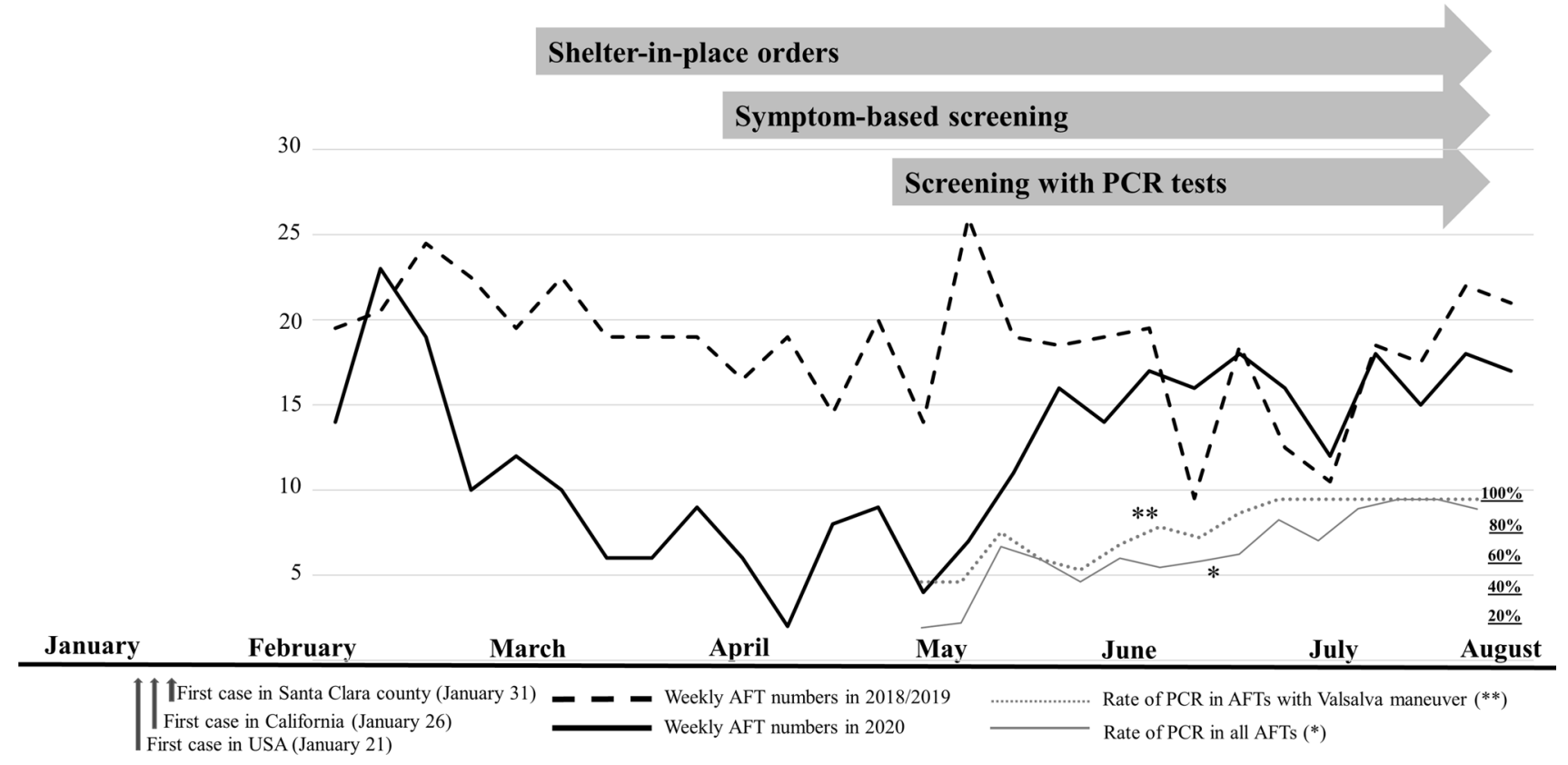

Fig. 1 A timeline of COVID-19 and weekly changes in AFT numbers. The weekly number of AFTs (solid line) declined due to community spread of COVID-19 and stay-at-home orders. With implement of the safety protocol, it has increased gradually back to similar

treating physician was notified. The patient became mildly symptomatic the day after the AFT. All potentially exposed HCWs remained asymptomatic with negative post-exposure PCR tests. From these two incidents, we learned that our protocol was causing confusion for the following reasons: (1) it was unclear whether PCR screening was recommended or mandatory and (2) for what AFT PCR screening was recommended or being required. Thus, we decided to streamline our protocol so that PCR tests no earlier than 7 days prior to AFT are required for all patients. Only $22.2 \%$ of patients and $50 \%$ of those with VM received PCR tests initially. The rate of PCR tests increased, reaching $100 \%$ within several weeks (Fig. 1). Among the 201 patients, 116 (57.7\%) were from neighboring counties (Santa Clara, San Francisco, San Mateo, Santa Cruz, Alameda), 76 (37.8\%) from other counties, and 9 (4.5\%) from other states. Rates of PCR tests did not differ between the neighboring counties and the other California counties (89/119 vs. 53/76; chi-square, $p=0.281$ ). The former group was more likely to have the tests done at Stanford (84/89 vs. 39/53; chi-square, $p<0.001)$. Patients were tested at Stanford 3 days (interquartile range: $2-5$ ) prior to AFTs and at other facilities 4 days (interquartile range: 3-7) prior (Mann-Whitney tests, $p=0.004)$. By late June, the number of AFTs performed had recovered compared to the past 2 years (Fig. 1). Twenty patients repeated PCR tests after AFTs for different reasons including pre-procedure screening or possible COVID-19. Half of these were done within 2 weeks of AFTs. All 20 patients had negative results. numbers from the past 2 years (dashed line). The two lines at the right lower corner show that the rate of PCR test as a screening tool has gone up to $100 \%$ over a few months in both all AFTs and AFTs with Valsalva maneuver

Lacking evidence-based, established safety guidance for AFT, Stanford autonomic center has implemented its own safety protocol to minimize transmission of COVID19. Through the protocol, we have not seen any COVID19 cases attributed to AFTs, although we cannot rule out possibilities of asymptomatic cases without repeated PCR tests. The PCR testing at our institution has specificity of 99.9\% and false-negative rate of $2.8 \%$ [3, 4]. When combined with testing at other facilities, they may differ, and infection risk during AFTs may change due to patients from other counties or states. Negative PCR results should not compromise the safety protocols, and HCWs should be aware of infection risks from asymptomatic patients. Our AFT safety protocol was instituted before the release of the AAS statement but has been modified several times based on HCW feedback and to reflect institutional policy updates. The current protocol is similar to the AAS statement guideline [1]. Differences include the use of PCR screening, using both disposable viral filter and tube during VM, and optional use of disposable gowns. This protocol may not be applicable at other centers in different situations [5]. From our experience, we learned that multidisciplinary efforts are critical, including those among HCWs, department, and infection control team. Clear and timely communication to the patients and their compliance are also key factors. 
Acknowledgements The authors would like to express sincere gratitude to Karenina Mijares-Blanco, Rose Mendoza, Erum Jahan, Timothy Fassler, and Aroonwan Thongkwan, who have provided patient care and support at our autonomic center during this difficult time.

\section{Compliance with ethical standards}

Conflict of interest The authors have nothing to disclose regarding this project. No conflict of interest.

\section{References}

1. Figueroa JJ, Cheshire WP, Claydon VE et al (2020) Autonomic function testing in the COVID-19 pandemic: an American Autonomic Society position statement. Clin Auton Res 30:295-297
2. The Center for Disease Constrol and Prevention (2020) Interim infection prevention and control recommendations for healthcare personnel during the Coronavirus disease 2019 (COVID-19) Pandemc. https://www.cdc.gov/coronavirus/2019-ncov/hcp/infectioncontrol-recommendations.html. (Accessed 26 Oct 2020)

3. Long DR, Gombar S, Hogan CA et al (2020) Occurrence and timing of subsequent SARS-CoV-2 RT-PCR positivity among initially negative patients. Clin Infect Dis. https://doi.org/10.1093/ cid/ciaa722

4. Boyd S, Kong C, Pinsky B, Banaei N (2020) Grand Rounds: COVID-19 Testing (PCR/Serology), Department of Medicine, Stanford University. https://www.youtube.com/watch?v=rd9kp T-TaJM\&t=948s. (Accessed 26 Oct 2020)

5. Guaraldi P, Barletta G, Baschieri C-B et al (2020) Testing cardiovascular autonomic function in the COVID-19 era: lessons from Bologna's autonomic unit. Clin Auton Res 30:325-330 